\title{
Morphological Evaluation of Metastatic Cholangiocarcinoma in a Dog
}

\author{
Andras-Laszlo NAGY*, Alexandru-Flaviu TABARAN, Marian MIHAIU, Cornel CĂTOI, Cosmina BOUARI, \\ Adrian OROS
}

Faculty of Veterinary Medicine, University of Agricultural Sciences and Veterinary Medicine, 3-5 Manastur street, 400372, Cluj-Napoca, Romania,

* Corresponding author e-mail: nagyandras26@gmail.com

Bulletin UASVM Veterinary Medicine 71(2) / 2014,

Print ISSN 1843-5270; Electronic ISSN 1843-5378

DOI:10.15835/buasvmcn-vm: 10542

\begin{abstract}
Cholangiocellular carcinomas are aggressive, malignant neoplasms of biliary epithelium usually arising from intrahepatic bile ducts. The aim of this study was to describe the immunohistochemical characteristics of metastatic ductular/papillary cholangiocarcinoma in a common breed dog. A 16-year old common breed dog was submitted for necropsy with an anamnesis of apathy, vomiting, anorexia and intermittent neurological symptoms. After complete necropsy, samples were taken and processed using paraffin technique. The sections were stained with Hematoxylin-eosin, Masson's trichrome, and PAS methods. Immunohistochemical examination was also performed using alpha smooth muscle actin, multicytokeratin AE1/AE3, cytokeratin 7, cytokeratin 19 and vimentin antibodies. At necropsy multiple masses in the liver parenchyma, and several metastases in the lungs, pancreas, intestinal serosa, omentum and on the surface of the diaphragm muscle were observed. Histologically the tumor consisted of pleomorphic epithelial cells, arranged in ductules, presenting intraluminal papillary growth pattern and marked fibroplasia. Vascular invasion, intra and extrahepatic metastases were also present. The tumoral cells were AE1/AE3, cytokeratin 7 and cytokeratin 19 positive, alfa smooth muscle actin and vimentin negative. Immunohistochemical exam proved to be useful in differentiating cholangiocellular carcinoma from hepatocellular carcinoma and several extrahepatic metastatic carcinomas.
\end{abstract}

Keywords: carcinoma, liver, tumor

Introduction. Primary hepatic tumors are rare in dogs, representing approximately $0.6-1.3 \%$ of canine neoplasms, and they include hepatocellular adenoma/carcinoma, cholangiocellular adenoma/carcinoma, mixed hepatocellular and cholangiocellular carcinomas, hepatic carcinoid and hepatoblastoma (Javanbakh tet al., 2013). Cholangiocellular carcinomas are aggressive, malignant neoplasms of the biliary epithelium and may occur as a large single mass, or as multiple tumors, with firm consistency, often showing central umbilication (Rothuijzen et al. 2006).

Materials and methods. A 16-year old common breed dog was submitted for necropsy with an anamnesis of apathy, vomiting, anorexia and intermittent neurological symptoms. After complete necropsy, samples were taken for histological examination. The samples were fixed in $10 \%$ buffered neutral formalin, and processed using paraffin technique. The slides were stained by Hematoxylin-eosin, Masson's trichrome, and PAS methods. Immunohistochemical examination was also performed using prediluted, ready to use antibodies (LeicaBond ${ }^{\mathrm{TM}}$,Leica Biosystems, Newcastle, United Kingdom), against alpha smooth muscle actin (Clone asm-1), multicytokeratin (Clone AE1/AE3), cytokeratin 7 (Clone RN7) cytokeratin 19 (Clone b170) and vimentin (Clone SRL33). Immunohistochemical study was realized using a Leica Bond-max ${ }^{\mathrm{TM}}$ immunohistochemistry system. 


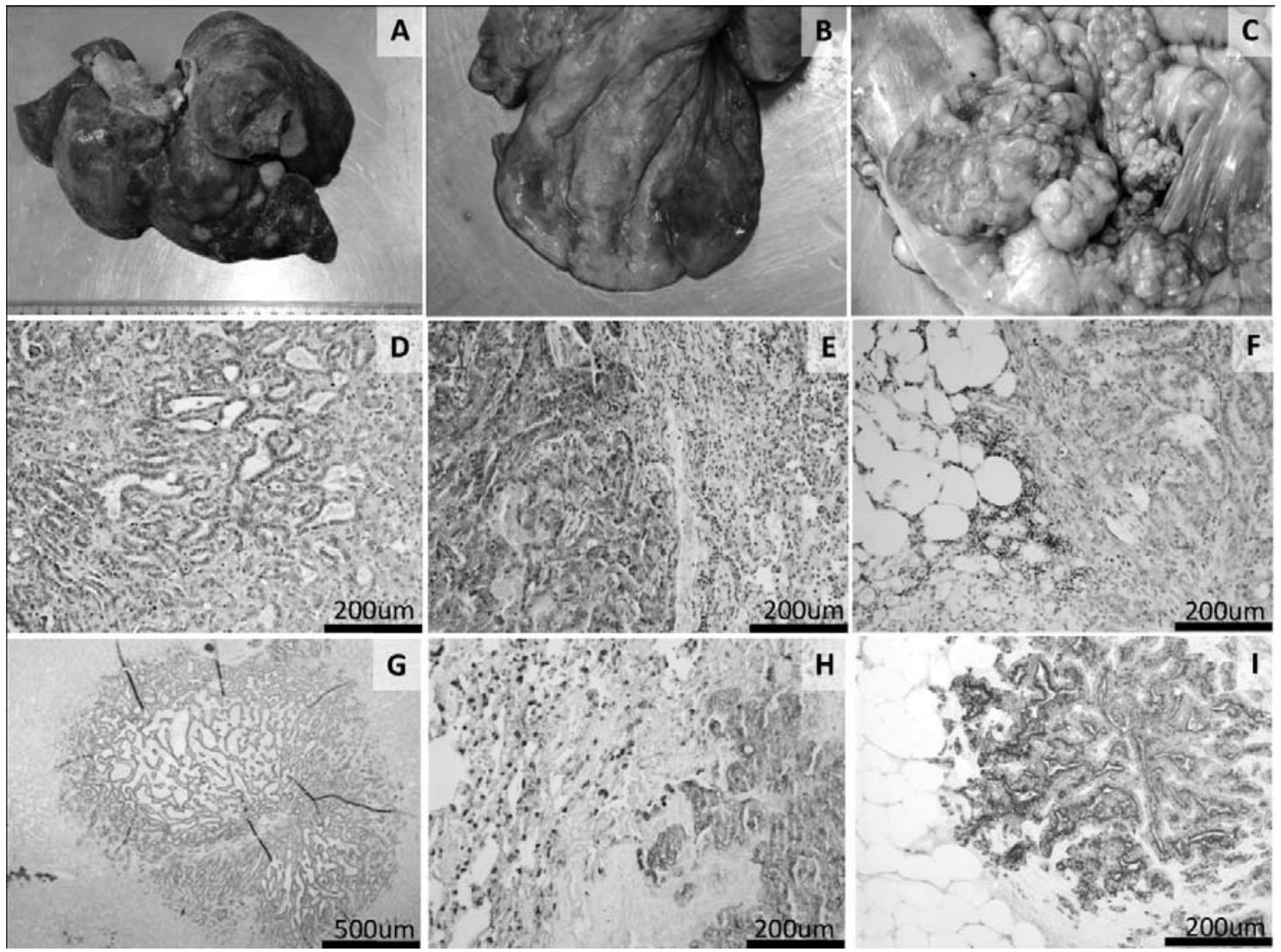

Fig.1. Multiple tumoral masses in the liver (A), lung (B) and omentum (C); tubular growth pattern of the neoplastic billiary epithelial cells (D), metastatic foci in the lung (E), metastatic foci in the omentum (F); Multicytokeratin positive tumoral cells in the liver (G), lung (H) and omentum (I).

Results and Discussion. At necropsy multiple masses with diameters ranging between $0,5-5 \mathrm{~cm}$ in the liver parenchyma, and several metastases in the lungs, pancreas, intestinal serosa, omentum and on the surface of the diaphragm muscle were observed. The liver masses, as well the metastases were scattered and multinodular with an umbilicated aspect (Fig.1A).

Histologically the tumor consisted of pleomorphic epithelial cells, arranged in ductules, presenting intraluminal papillary growth pattern and marked fibroplasia (Fig.1A). Vascular invasion, intra and extrahepatic metastases were present, having the same histologic characteristics with the primary tumor (Fig.1E,F). The tumoral cells were AE1/AE3, cytokeratin 7 and cytokeratin 19 positive, alfa smooth muscle actin and vimentin negative, confirming the diagnosis of cholangiocarcinoma (Fig.1G,H,I).
Conclusion. Immunohistochemical exam is useful in differentiating cholangiocellular carcinoma from hepatocellular carcinoma and several extrahepatic metastatic carcinomas.

Acknowledgement. This paper was published under the frame of European Social Found, Human Resources Development Operational Programme 2007-2013, projectno. POSDRU/159/1.5/S/136893.

\section{References}

1. Javanbakht J, Sasani F, Khaki F, Jamshidi S, Hassan MA, et al. (2013) Evaluation of Metastatic Cholangiocarcinoma in a Spitz Dog. J Cancer Sci Ther 5: 113-114.

2. Rothuizen J, Bunch SE, Charles JA, Cullen JM, Desmet VJ, et al. (2006) WSAVA Standards for Clinical and Histological Diagnosis of Canine and Feline Liver Disease. Philadelphia, PA, USA: Saunders Elsevier, 41-117. 